Pacific

Journal of

Mathematics

\title{
ON THE STEINBERG CHARACTER OF
}

A SEMISIMPLE $p$-ADIC GROUP

Ju-LeE Kim AND GeORge LuSZTiG

Volume 265 No. 2

October 2013 


\title{
ON THE STEINBERG CHARACTER OF A SEMISIMPLE $p$-ADIC GROUP
}

\author{
Ju-LeE Kim AND GeORGe LuszTiG
}

Dedicated to Robert Steinberg on the occasion of his ninetieth birthday.

\begin{abstract}
We show that the character of the Steinberg representation of a split semisimple p-adic group at a very regular element is given (up to sign) by a power of $q$, the number of elements in the residue field. We also show that (under an assumption on the characteristic) the character of an Iwahorispherical representation at a split very regular element is given by a trace in the corresponding Hecke algebra module.
\end{abstract}

\section{Introduction}

1.1. Let $K$ be a nonarchimedean local field and let $\underline{K}$ be a maximal unramified field extension of $K$. Let $\mathcal{O}$ be the ring of integers of $K$ and let $\mathfrak{p}$ be the maximal ideal of $\mathcal{O}$; the counterparts for $\underline{K}$ are denoted by $\underline{\mathcal{O}}$ and $\mathfrak{p}$. Let $\underline{K}^{*}=\underline{K}-\{0\}$. We write $\mathcal{O} / \mathfrak{p}=F_{q}$, a finite field with $q$ elements of characteristic $p$.

Let $G$ be a semisimple almost simple algebraic group defined and split over $K$ with a given $\mathcal{O}$-structure compatible with the $K$-structure.

If $V$ is an admissible representation of $G(K)$ of finite length, we denote by $\phi_{V}$ the character of $V$ in the sense of Harish-Chandra, viewed as a $\mathbb{C}$-valued function on the set $G(K)_{r s}:=G_{r s} \cap G(K)$. (Here, $G_{r s}$ is the set of regular semisimple elements of $G$, and $\mathbb{C}$ is the field of complex numbers.)

In this paper we study the restriction of the function $\phi_{V}$ to:

(a) a certain subset $G(K)_{v r}$ of $G(K)_{r s}$, namely, the set of very regular elements in $G(K)$ (see 1.2) in the case where $V$ is the Steinberg representation of $G(K)$, and

(b) a certain subset $G(K)_{s v r}$ of $G(K)_{v r}$, namely, the set of split very regular elements in $G(K)$ (see 1.2) in the case where $V$ is an irreducible admissible representation of $G(K)$ with nonzero vectors fixed by an Iwahori subgroup.

Both authors are supported in part by the National Science Foundation.

MSC2010: 20G99.

Keywords: $p$-adic group, character, unipotent representation. 
In case (a), we show that $\phi_{V}(g)$ with $g \in G(K)_{r s}$ is of the form $\pm q^{n}$ with $n \in\{0,-1,-2, \ldots\}$ (see Corollary 3.4) with more precise information when $g \in G(K)_{s v r}$ (see Theorem 2.2) or when $g \in G(K)_{c v r}$ (see Theorem 3.2). In case (b) we show (with some restriction on characteristic) that $\phi_{V}(g)$ with $g \in G(K)_{s v r}$ can be expressed as the trace of a certain element of an affine Hecke algebra on an irreducible module (see Theorem 4.3).

Note that the Steinberg representation $\mathbf{S}$ is an irreducible admissible representation of $G(K)$ with a one-dimensional subspace invariant under an Iwahori subgroup on which the corresponding affine Hecke algebra acts through the "sign" representation; see [Matsumoto 1969; Shalika 1970]. This is a $p$-adic analogue of the Steinberg representation [Steinberg 1951] of a reductive group over $F_{q}$. In [Rodier 1986], it is proven that $\phi_{\mathbf{S}}(g) \neq 0$ for any $g \in G(K)_{r s}$.

1.2. Let $g \in G_{r s} \cap G(\underline{K})$. Let $T^{\prime}=T_{g}^{\prime}$ be the maximal torus of $G$ that contains $g$. We say that $g$ is very regular if $T^{\prime}$ is split over $\underline{K}$ and for any root $\alpha$ with respect to $T^{\prime}$ viewed as a homomorphism $T^{\prime}(\underline{K}) \rightarrow \underline{K}^{*}$ we have $\alpha(g) \notin(1+\mathfrak{p})$. If, in addition, $\alpha(g) \in \underline{\mathcal{O}}$, we say that $g$ is compact very regular.

Let $G(\underline{K})_{v r}$ be the set of elements in $G(\underline{K})$ that are very regular, and $G(\underline{K})_{c v r}$ the set of compact very regular ones. We write $G(K)_{v r}=G(\underline{K})_{v r} \cap G(K)$ and $G(K)_{c v r}=G(\underline{K})_{c v r} \cap G(K)$. Let $G(K)_{s v r}$ be the set of all $g \in G(K)_{v r}$ such that $T_{g}^{\prime}$ is split over $K$.

1.3. Notation. Let $K^{*}=K-\{0\}$, and let $v: K^{*} \rightarrow \mathbb{Z}$ be the unique (surjective) homomorphism such that $v\left(\mathfrak{p}^{n}-\mathfrak{p}^{n+1}\right)=n$ for any $n \in \mathbb{N}$. For $a \in K^{*}$ we set $|a|=q^{-v(a)}$.

We fix a maximal torus $T$ of $G$ defined and split over $K$. Let $Y$ (resp. $X$ ) be the group of cocharacters (resp. characters) of the algebraic group $T$. Let $\langle\rangle:, Y \times X \rightarrow \mathbb{Z}$ be the obvious pairing. Let $R \subset X$ be the set of roots of $G$ with respect to $T$, let $R^{+}$be a set of positive roots for $R$, and let $\Pi$ be the set of simple roots of $R$ determined by $R^{+}$. We write $\Pi=\left\{\alpha_{i}: i \in I\right\}$. Let $R^{-}=R-R^{+}$. Let $Y^{+}$(resp. $Y^{++}$) be the set of all $y \in Y$ such that $\langle y, \alpha\rangle \geq 0$ (resp. $\langle y, \alpha\rangle>0$ ) for all $\alpha \in R^{+}$. We define $2 \rho \in X$ by $2 \rho=\sum_{a \in R^{+}} \alpha$.

We have canonically $T(K)=K^{*} \otimes Y$; we define a homomorphism $\chi: T(K) \rightarrow Y$ by $\chi(\lambda \otimes y)=v(\lambda) y$ for any $\lambda \in K^{*}, y \in Y$. For any $y \in Y$, we set $T(K)_{y}=$ $\chi^{-1}(y)$. For $y \in Y$, let $T(K)_{y}^{\natural}=T(K)_{y} \cap G(K)_{s v r}$. Note that if $y \in Y^{++}$then $T(K)_{y}^{\natural}=T(K)_{y}$.

For each $\alpha \in R$ let $U_{\alpha}$ be the corresponding root subgroup of $G$.

\section{Calculation of $\phi_{\mathrm{S}}$ on $G(K)_{s v r}$}

2.1. Let $\mathcal{W} \subset \operatorname{Aut}(T)$ be the Weyl group of $G$ regarded as a Coxeter group; for $i \in I$, let $s_{i}$ be the simple reflection in $\mathcal{W}$ determined by $\alpha_{i}$. We can also view 
$\mathcal{W}$ as a subgroup of $\operatorname{Aut}(Y)$ or $\operatorname{Aut}(X)$. Let $w=w_{0}$ be the longest element of $\mathcal{W}$. For any $J \subset I$, let $\mathcal{W}_{J}$ be the subgroup of $\mathcal{W}$ generated by $\left\{s_{i}: i \in J\right\}$ and let $R_{J}=R \cap \sum_{i \in J} \mathbb{Z} \alpha_{i}$. Let

$$
R_{J}^{+}=R_{J} \cap R^{+} \quad \text { and } \quad R_{J}^{-}=R_{J}-R_{J}^{+} .
$$

Let $\mathfrak{g}$ be the Lie algebra of $G$, and let $\mathfrak{t} \subset \mathfrak{g}$ be the Lie algebra of $T$. For any $J \subset I$, let $\mathfrak{l}_{J}$ be the Lie subalgebra of $\mathfrak{g}$ spanned by $\mathfrak{t}$ and the root spaces corresponding to the roots in $R_{J}$. Let $\mathfrak{n}_{J}$ be the Lie subalgebra of $\mathfrak{g}$ spanned by the root spaces corresponding to roots in $R^{+}-R_{J}^{+}$.

According to [Casselman 1973], $\phi_{\mathbf{S}}$ is an alternating sum of characters of representations induced from one-dimensional representations of various parabolic subgroups of $G$ defined over $K$. From this, one can deduce that if $t \in T(K) \cap G(K)_{r s}$ then

$$
\phi_{\mathbf{S}}(t)=\sum_{J \subset I}(-1)^{\sharp J} \sum_{w \in J} \delta_{J}(w(t))^{1 / 2} D_{I, J}(w(t))^{-1 / 2},
$$

where for any $J \subset I$ and $t^{\prime} \in T(K) \cap G(K)_{r s}$ we set

$$
\begin{aligned}
D_{I, J}\left(t^{\prime}\right) & =\left|\operatorname{det}\left(1-\left.\operatorname{Ad}\left(t^{\prime}\right)\right|_{\mathfrak{g} / \mathfrak{l}_{J}}\right)\right|, \\
\delta_{J}\left(t^{\prime}\right) & =\left|\operatorname{det}\left(\left.\operatorname{Ad}\left(t^{\prime}\right)\right|_{\mathfrak{n}_{J}}\right)\right|,
\end{aligned}
$$

and ${ }^{J} \mathcal{W}$ is the set of representatives of minimal length for the cosets $\mathcal{W}_{J} \backslash \mathcal{W}$. Here for a real number $a \geq 0$ we denote by $a^{1 / 2}$ or $\sqrt{a}$ the nonnegative square root of $a$. Writing $\phi$ instead of $\phi_{\mathbf{S}}$, we have:

Theorem 2.2. Let $y \in Y^{+}$and let $t \in T(K)_{y}^{\natural}$. Then $\phi(t)=q^{-\langle y, 2 \rho\rangle}$.

2.3. More generally, let $t \in T(K)_{y}^{\mathfrak{a}}$, where $y \in Y$. By a standard property of Weyl chambers, there exists $w \in \mathcal{W}$ such that $w(y) \in Y^{+}$. Let $t_{1}=w(t)$. Then the theorem is applicable to $t_{1}$, and we have $\phi(t)=\phi\left(t_{1}\right)=q^{-\langle w(y), 2 \rho\rangle}$.

2.4. Let $y^{\prime}=w_{0}(y), t^{\prime}=w_{0}(t)$. We have $\phi_{\mathbf{S}}(t)=\phi_{\mathbf{S}}\left(t^{\prime}\right), t^{\prime} \in T(K)_{y^{\prime}},-y^{\prime} \in Y^{+}$. We show that

$$
v\left(1-\beta\left(t^{\prime}\right)\right)= \begin{cases}v\left(\beta\left(t^{\prime}\right)\right) & \text { if } \beta \in R^{+}, \\ 0 & \text { if } \beta \in R^{-} .\end{cases}
$$

Assume first that $\beta \in R^{+}$. If $v\left(\beta\left(t^{\prime}\right)\right) \neq 0$ then $v\left(\beta\left(t^{\prime}\right)\right)<0$ (since $\left\langle y^{\prime}, \beta\right\rangle \neq 0$ and $\left.\left\langle y^{\prime}, \beta\right\rangle \leq 0\right)$; hence, $v\left(1-\beta\left(t^{\prime}\right)\right)=v\left(\beta\left(t^{\prime}\right)\right)$. If $v\left(\beta\left(t^{\prime}\right)\right)=0$ then $\beta\left(t^{\prime}\right)-1 \in \mathcal{O}-\mathfrak{p}$; hence, $v\left(1-\beta\left(t^{\prime}\right)\right)=0=v\left(\beta\left(t^{\prime}\right)\right)$ as required.

Assume next that $\beta \in R^{-}$. If $v\left(\beta\left(t^{\prime}\right)\right) \neq 0$ then $v\left(\beta\left(t^{\prime}\right)\right)>0$ (since $\left\langle y^{\prime}, \beta\right\rangle \neq 0$ and $\left.\left\langle y^{\prime}, \beta\right\rangle \geq 0\right)$; hence, $v\left(1-\beta\left(t^{\prime}\right)\right)=0$. If $v\left(\beta\left(t^{\prime}\right)\right)=0$ then $\beta\left(t^{\prime}\right)-1 \in \mathcal{O}-\mathfrak{p}$; hence, $v\left(1-\beta\left(t^{\prime}\right)\right)=0$ as required. 
For any $w \in \mathcal{W}, J \subset I$ we have

$$
D_{I, J}\left(w\left(t^{\prime}\right)\right)=\prod_{\alpha \in R-R_{J}} q^{-v\left(1-\alpha\left(w\left(t^{\prime}\right)\right)\right)}=\prod_{\substack{\alpha \in R-R_{J} \\ w^{-1} \alpha \in R^{+}}} q^{-v\left(\alpha\left(w\left(t^{\prime}\right)\right)\right)}=\prod_{\substack{\alpha \in R-R_{J} \\ w^{-1} \alpha \in R^{+}}} q^{-\left\langle y^{\prime}, w^{-1} \alpha\right\rangle}
$$

and

$$
\delta_{J}\left(w\left(t^{\prime}\right)\right)=\prod_{\alpha \in R^{+}-R_{J}^{+}} q^{-v\left(\alpha\left(w\left(t^{\prime}\right)\right)\right)}=\prod_{\alpha \in R^{+}-R_{J}^{+}} q^{-\left\langle y^{\prime}, w^{-1} \alpha\right\rangle} .
$$

(We have used (1) with $\beta=w^{-1}(\alpha)$.) We see that

$$
\phi(t)=\phi\left(t^{\prime}\right)=\sum_{J \subset I}(-1)^{\sharp J} \sum_{w \in \in^{J} \mathcal{W}} \sqrt{q}^{-\left\langle y^{\prime}, x_{w, J}\right\rangle},
$$

where for $w \in{ }^{J} \mathcal{W}$ we have

$$
\begin{aligned}
x_{w, J}= & \sum_{\alpha \in R^{+}-R_{J}^{+}} w^{-1} \alpha-\sum_{\substack{\alpha \in R-R_{J} \\
w^{-1} \alpha \in R^{+}}} w^{-1} \alpha \\
= & \sum_{\substack{\alpha \in R^{+}-R_{J}^{+} \\
w^{-1}(\alpha) \in R^{-}}} w^{-1} \alpha-\sum_{\substack{\alpha \in R^{-}-R_{J}^{-} \\
w^{-1}(\alpha) \in R^{+}}} w^{-1} \alpha \\
= & 2 \sum_{\substack{\alpha \in R^{+}-R_{J}^{+} \\
w^{-1} \alpha \in R^{-}}} w^{-1} \alpha \in X .
\end{aligned}
$$

For $w \in{ }^{J} \mathcal{W}$, we have $\alpha \in R_{J}^{+} \Rightarrow w^{-1} \alpha \in R^{+}$; hence,

$$
\sum_{\substack{\alpha \in R^{+}-R_{J}^{+} \\ w^{-1} \alpha \in R^{-}}} w^{-1} \alpha=\sum_{\substack{\alpha \in R^{+} \\ w^{-1} \alpha \in R^{-}}} w^{-1} \alpha,
$$

so that $x_{w, J}=x_{w}$, where

Thus, we have

$$
x_{w}=2 \sum_{\substack{\alpha \in R^{+} \\ w^{-1} \alpha \in R^{-}}} w^{-1} \alpha \in X
$$

$$
\phi(t)=\sum_{J \subset I}(-1)^{\sharp J} \sum_{w \in \mathcal{J}^{J} \mathcal{W}} \sqrt{q}^{-\left\langle y^{\prime}, x_{w}\right\rangle}=\sum_{w \in \mathcal{W}} c_{w} \sqrt{q}^{-\left\langle y^{\prime}, x_{w}\right\rangle},
$$

where for $w \in \mathcal{W}$ we set

$$
c_{w}=\sum_{\substack{J \subset I \\ w \in{ }^{J} \mathcal{W}}}(-1)^{\sharp J} .
$$

For $w \in \mathcal{W}$, let $\mathcal{L}(w)=\left\{i \in I: s_{i} w>w\right\}$, where $>$ refers to the standard partial 
order on $\mathcal{W}$. For $J \subset I$, we have $w \in{ }^{J} \mathcal{W}$ if and only if $J \subset \mathcal{L}(w)$; thus,

$$
c_{w}=\sum_{J \subset \mathcal{L}(w)}(-1)^{\sharp J},
$$

and this is 0 unless $\mathcal{L}(w)=\varnothing$ (that is $w=w_{0}$ ), in which case $c_{w}=1$. Note also that $x_{w_{0}}=-4 \rho$; thus, we have

$$
\phi(t)=c_{w_{0}} \sqrt{q}^{-\left\langle y^{\prime}, x_{w_{0}}\right\rangle}=q^{\left\langle y^{\prime}, 2 \rho\right\rangle}=q^{-\langle y, 2 \rho\rangle} .
$$

Theorem 2.2 is proved.

2.5. Assume now that $\tau \in T(K)$ satisfies the following condition: for any $\alpha \in R$ we have $\alpha(\tau)-1 \in \mathfrak{p}-\{0\}$ so that $\alpha(\tau)-1 \in \mathfrak{p}^{n_{\alpha}}-\mathfrak{p}^{n_{\alpha}+1}$ for a well defined integer $n_{\alpha} \geq 1$. Note that $n_{-\alpha}=n_{\alpha}$ and $v(1-\alpha(\tau))=n_{\alpha} \geq 1$ for all $\alpha \in R$; hence,

$$
\phi(\tau)=\sum_{J \subset I}(-1)^{\sharp J} \sum_{w \in J} q^{\sum_{\alpha \in R} n_{\alpha} / 2-\sum_{\alpha \in R_{J}} n_{w^{-1}(\alpha)} / 2} .
$$

Thus,

$$
\phi(\tau)=\sharp(\mathcal{W}) q^{\sum_{\alpha \in R} n_{\alpha} / 2}+\text { strictly smaller powers of } q .
$$

In the case where $K$ is the field of power series over $F_{q}$, the leading term in (2) is equal to $\sharp(\mathcal{W}) q^{m}$, where $m$ is the dimension of the "variety" of Iwahori subgroups of $G(\underline{K})$ that contain the topologically unipotent element $\tau$ (see [Kazhdan and Lusztig 1988]).

\section{Calculation of $\phi_{\mathrm{S}}$ on $G(K)_{v r}$}

3.1. We will again write $\phi$ instead of $\phi_{\mathbf{S}}$. In this section we assume that we are given $\gamma \in G(K)_{v r}$. Let $T^{\prime}=T_{\gamma}^{\prime}$. Note that $T^{\prime}$ is defined over $K$; let $A^{\prime}$ be the largest $K$-split torus of $T^{\prime}$. For any parabolic subgroup $P$ of $G$ defined over $K$ such that $\gamma \in P$, we set $\delta_{P}(\gamma)=\left|\operatorname{det}\left(\left.\operatorname{Ad}(\gamma)\right|_{\mathfrak{n}}\right)\right|$, where $\mathfrak{n}$ is the Lie algebra of the unipotent radical of $P$.

Let $\mathcal{X}$ be the set of all pairs $(P, A)$, where $P$ is a parabolic subgroup of $G$ defined over $K$ and $A$ is the unique maximal $K$-split torus in the center of some Levi subgroup of $P$ defined over $K$. Then that Levi subgroup is uniquely determined by $A$ and is denoted by $M_{A}$. Let $\mathcal{X}^{\prime}=\left\{(P, A) \in \mathcal{X}: A \subset A^{\prime}\right\}$. According to [HarishChandra 1973], we have

$$
\phi(\gamma)=(-1)^{\operatorname{dim} T} \sum_{(P, A) \in \mathcal{X}^{\prime}}(-1)^{\operatorname{dim} A} \delta_{P}(\gamma)^{1 / 2} D_{G / M_{A}}(\gamma)^{-1 / 2},
$$

where $D_{G / M_{A}}(\gamma)=\mid \operatorname{det}\left(1-\left.\operatorname{Ad}(\gamma)\right|_{\mathfrak{g} / \mathfrak{l})}\right)$ (we denote by $\mathfrak{l}$ the Lie algebra of $\left.M_{A}\right)$.

Theorem 3.2. Assume in addition that $\gamma \in G(K)_{c v r}$. Then $\phi(\gamma)=(-1)^{\operatorname{dim} T-\operatorname{dim} A^{\prime}}$. 
Proof. From our assumptions we see that $\delta_{P}(\gamma)=1=D_{G / M_{A}}(\gamma)$ for all $(P, A) \in \mathcal{X}^{\prime}$; hence, (3) becomes

$$
\phi(\gamma)=(-1)^{\operatorname{dim} T} \sum_{(P, A) \in \mathcal{X}^{\prime}}(-1)^{\operatorname{dim} A} .
$$

Let $\mathcal{Y}$ be the group of cocharacters of $A^{\prime}$ and let $\mathfrak{H}=\mathcal{Y} \otimes \mathbb{R}$. The real vector space $\mathfrak{H}$ can be partitioned into facets $F_{P, A}$ indexed by $(P, A) \in \mathcal{X}^{\prime}$ such that $F_{P, A}$ is homeomorphic to $\mathbb{R}^{\operatorname{dim} A}$. Note that the Euler characteristic with compact support of $F_{P, A}$ is $(-1)^{\operatorname{dim} A}$, and the Euler characteristic with compact support of $\mathfrak{H}$ is $(-1)^{\operatorname{dim}_{\mathbb{R}} \mathfrak{H}}=(-1)^{\operatorname{dim} A^{\prime}}$. Using the additivity of the Euler characteristic with compact support we see that $\sum_{(P, A) \in \mathcal{X}^{\prime}}(-1)^{\operatorname{dim} A}=(-1)^{\operatorname{dim} A^{\prime}}$; thus, $\phi(\gamma)=$ $(-1)^{\operatorname{dim} T-\operatorname{dim} A^{\prime}}$, as required.

3.3. In the setup of 3.1 , let $P_{\gamma}$ be the parabolic subgroup of $G$ associated to $\gamma$ as in [Casselman 1977]. Note that $P_{\gamma}$ is defined over $K$. The following result can be deduced by combining Theorem 3.2 with the results in [Casselman 1977] and with Proposition 2 in [Rodier 1986].

Corollary 3.4. We have $\phi(\gamma)=(-1)^{\operatorname{dim} T-\operatorname{dim} A^{\prime}} \delta_{P_{\gamma}}(\gamma)$.

The corollary provides another proof of Theorem 2.2.

\section{Iwahori spherical representations: split elements}

4.1. Let $B$ be the subgroup of $G(K)$ generated by

$$
\left\{U_{\alpha}(\mathcal{O}): \alpha \in R^{+}\right\} \cup\left\{U_{\alpha}(\mathfrak{p}): \alpha \in R^{-}\right\} \cup T(K)_{0} .
$$

(The subgroups $U_{\alpha}(\mathcal{O}), U_{\alpha}(\mathfrak{p})$ of $U_{\alpha}$ are defined by the $\mathcal{O}$-structure of $G$.) Then $B$ is an Iwahori subgroup of $G(K)$. For any $\alpha \in R$ we choose an isomorphism $x_{\alpha}: K \stackrel{\sim}{\rightarrow} U_{\alpha}(K)$ (the restriction of an isomorphism of algebraic groups from the additive group to $U_{\alpha}$ ), which carries $\mathcal{O}$ onto $U_{\alpha}(\mathcal{O})$ and $\mathfrak{p}$ onto $U_{\alpha}(\mathfrak{p})$. We set $W:=Y \cdot \mathcal{W}$ with $Y$ normal in $W$ (recall that $\mathcal{W}$ acts naturally on $Y$ ). Let $Y^{\prime}$ be the subgroup of $Y$ generated by the coroots. Then $W^{\prime}:=Y^{\prime} \cdot \mathcal{W}$ is naturally a subgroup of $W$. According to [Iwahori and Matsumoto 1965], $W$ is an extended Coxeter group (the semidirect product of the Coxeter group $W^{\prime}$ with the finite abelian group $Y / Y^{\prime}$ ) with length function

$$
l(y w)=\sum_{\substack{\alpha \in R^{+} \\ w^{-1}(\alpha) \in R^{+}}}\|\langle y, \alpha\rangle\|+\sum_{\substack{\alpha \in R^{+} \\ w^{-1}(\alpha) \in R^{-}}}\|\langle y, \alpha\rangle-1\|,
$$

where $\|a\|=a$ if $a \geq 0$ and $\|a\|=-a$ if $a<0$. From the same reference we know that the set of double cosets $B \backslash G(K) / B$ is in bijection with $W$; to $y w$ (where $y \in Y, w \in \mathcal{W}$ ) corresponds the double coset $\Omega_{y w}$ containing $T(K)_{y} \dot{w}$ (here $\dot{w}$ is 
an element in $G(\mathcal{O})$ which normalizes $T(K)_{0}$ and acts on it in the same way as $w$ ); moreover, $\sharp\left(\Omega_{y w} / B\right)=\sharp\left(B \backslash \Omega_{y w}\right)=q^{l(y w)}$ for any $y \in Y, w \in \mathcal{W}$. For example, if $y \in Y^{++}$then $l(y)=\langle y, 2 \rho\rangle$.

Let $H$ be the algebra of $B$-biinvariant functions $G(K) \rightarrow \mathbb{C}$ with compact support with respect to convolution (we use the Haar measure $d g$ on $G(K)$ for which $\operatorname{vol}(B)=1)$. For $y, w$ as above, let $\mathfrak{T}_{y w} \in H$ be the characteristic function of $\Omega_{y w}$. Then the functions $\mathfrak{T}_{\underline{w}}, \underline{w} \in W$ form a $\mathbb{C}$-basis of $H$, and according to [Iwahori and Matsumoto 1965], we have

$$
\begin{aligned}
\mathfrak{T}_{\underline{w}} \mathfrak{T}_{\underline{w}^{\prime}}=\mathfrak{T}_{\underline{w} \underline{w}^{\prime}} & \text { for } \underline{w}, \underline{w}^{\prime} \in W \text { with } l\left(\underline{w} \underline{w}^{\prime}\right)=l(\underline{w})+l\left(\underline{w}^{\prime}\right), \\
\left(\mathfrak{T}_{\underline{w}}+1\right)\left(\mathfrak{T}_{\underline{w}}-q\right)=0 & \text { for } \underline{w} \in W^{\prime} \text { with } l(\underline{w})=1 .
\end{aligned}
$$

In other words, $H$ is what one now calls the Iwahori-Hecke algebra of the (extended) Coxeter group $W$ with parameter $q$.

4.2. Let $\mathcal{C}_{0}^{\infty}(G(K))$ be the vector space of locally constant functions with compact support from $G(K)$ to $\mathbb{C}$. Let $(V, \sigma)$ be an irreducible admissible representation of $G(K)$ such that the space $V^{B}$ of $B$-invariant vectors in $V$ is nonzero. If $f \in$ $\mathcal{C}_{0}^{\infty}(G(K))$ then there is a well defined linear map $\sigma_{f}: V \rightarrow V$ such that for any $x \in V$ we have $\sigma_{f}(x)=\int_{G} f(g) \sigma(g)(x) d g$. This linear map has finite rank; hence, it has a well defined $\operatorname{trace} \operatorname{tr}\left(\sigma_{f}\right) \in \mathbb{C}$. From the definitions we see that for $f, f^{\prime} \in \mathcal{C}_{0}^{\infty}(G(K))$ we have $\sigma_{f * f^{\prime}}=\sigma_{f} \sigma_{f^{\prime}}: V \rightarrow V$ where $*$ denotes convolution. If $f \in H$ then $\sigma_{f}$ maps $V$ into $V^{B}$ and $\operatorname{tr}\left(\sigma_{f}\right)=\operatorname{tr}\left(\left.\sigma_{f}\right|_{V^{B}}\right)$. (Recall that $\operatorname{dim} V^{B}<\infty$.) We see that the maps $\left.\sigma_{f}\right|_{V^{B}}$ define a (unital) $H$-module structure on $V^{B}$. It is known that the $H$-module $V^{B}$ is irreducible [Borel 1976]. Moreover, for $\underline{w} \in W$ we have $\operatorname{tr}\left(\sigma_{\mathfrak{T}_{\underline{w}}}\right)=\operatorname{tr}\left(\mathfrak{T}_{\underline{w}}\right)$, where the trace in the right side is taken in the $\bar{H}$-module $V^{B}$.

Theorem 4.3. Assume that $K$ has characteristic zero and that $p$ is sufficiently large. Let $y \in Y^{+}$and $t \in T(K)_{y}$. We have

$$
\phi_{V}(t)=q^{-\langle y, 2 \rho\rangle} \operatorname{tr}\left(\mathfrak{T}_{y}\right),
$$

where the trace in the right side is taken in the irreducible $H$-module $V^{B}$.

An equivalent statement is that

$$
\phi_{V}(t)=\operatorname{tr}\left(\sigma_{\mathfrak{T}_{y}}\right) / \operatorname{vol}\left(\Omega_{y}\right) .
$$

(Recall that $\mathfrak{T}_{y}$ on the right side is the characteristic function of $\Omega_{y}=B T(K)_{y} B$.)

The assumption on characteristic in the theorem is needed only to be able to use a result from [Adler and Korman 2007]; see (5) below. We expect that the theorem holds without that assumption.

In the case where $y=0$, the theorem becomes

$$
t \in T(K) \cap G_{c v r} \Longrightarrow \phi_{V}(t)=\operatorname{dim}\left(V^{B}\right) .
$$


As pointed out to us by R. Bezrukavnikov and S. Varma, in the special case where $y \in Y^{++}$, Theorem 4.3 can be deduced from results in [Casselman 1977].

4.4. In the case where $V=\mathbf{S}$ (see 1.1), for any $y \in Y^{+}, \mathfrak{T}_{y}$ acts on the onedimensional vector space $V^{B}$ as the identity map, so that $\phi_{V}(t)=q^{-\langle y, 2 \rho\rangle}$ for all $t \in T(K)_{y}$. We thus recover Theorem 2.2 (which holds in any characteristic).

\section{Proof of Theorem 4.3}

5.1. Let $B=B_{0}, B_{1}, B_{2}, \ldots$ be the strictly decreasing Moy-Prasad [1994] filtration of $B$. This is a sequence associated to a point $x$ in the building such that $B=G_{x, 0}$. Each $B_{i} / B_{i+1}$ is abelian. Let $T_{n}:=T(K) \cap B_{n}$. Applying [Adler and Korman 2007, Corollary 12.11] to $\phi_{V}$, we conclude that

$$
\phi_{V} \text { is constant on the } \operatorname{Ad}(G) \text {-orbit }{ }^{G}\left(t T_{1}\right) \text { of } t T_{1} \text {. }
$$

Lemma 5.2. Let $n \geq 1$. For any $t^{\prime} \in T(K)_{y}^{\bullet}$ and $z \in B_{n}$, there exist $g \in B_{n}, t^{\prime \prime} \in T_{n}$, and $z^{\prime} \in B_{n+1}$ such that $\operatorname{Ad}(g)\left(t^{\prime} z\right)=t^{\prime} t^{\prime \prime} z^{\prime}$.

Proof. Let $Z=\left\{\alpha \in R: U_{\alpha} \cap B_{n} \supsetneq U_{\alpha} \cap B_{n+1}\right\}$. If $Z=\varnothing$ then $B_{n}=T_{n} B_{n+1}$; hence, $z=t^{\prime \prime} z^{\prime}$ for some $t^{\prime \prime} \in T_{n}$ and $z^{\prime} \in B_{n+1}$, and one can take $g=1$. If $Z \neq \varnothing$ then we can find $a_{\alpha} \in K$ for each $\alpha \in Z$ such that $x_{\alpha}\left(a_{\alpha}\right) \in B_{n}$ and $z \equiv \prod_{\alpha \in Z} x_{\alpha}\left(a_{\alpha}\right)\left(\bmod T_{n} B_{n+1}\right)$. Such $a_{\alpha}$ can be chosen independent of the order of the product since $B_{n} / T_{n} B_{n+1}$ is abelian. Take $g=\prod_{\alpha \in Z} x_{\alpha}\left(\left(1-\alpha\left(t^{\prime-1}\right)\right)^{-1} a_{\alpha}\right)$. Then $g \in B_{n}$ since $\left|1-\alpha\left(t^{\prime-1}\right)\right| \geq 1$ for $y \in Y^{+}$. (To show $\left|1-\alpha\left(t^{\prime-1}\right)\right| \geq 1$ for $y \in Y^{+}$, we argue as for (1). Assume first that $\alpha \in R^{+}$. If $v\left(\alpha\left(t^{\prime-1}\right)\right) \neq 0$ then $v\left(\alpha\left(t^{\prime-1}\right)\right)<0$ (since $\langle y, \alpha\rangle \neq 0,\langle y, \alpha\rangle \geq 0$ ); therefore, $v\left(1-\alpha\left(t^{\prime-1}\right)\right)=v\left(\alpha\left(t^{\prime-1}\right)\right)<0$ and $\left|1-\alpha\left(t^{\prime-1}\right)\right|>1$. If $v\left(\alpha\left(t^{\prime-1}\right)\right)=0$ then $\alpha\left(t^{\prime-1}\right)-1 \in \mathcal{O}-\mathfrak{p}$; hence, $v\left(1-\alpha\left(t^{\prime-1}\right)\right)=0$ and $\left|1-\alpha\left(t^{\prime-1}\right)\right|=1$ as required. Assume next that $\alpha \in R^{-}$. If $v\left(\alpha\left(t^{\prime-1}\right)\right) \neq 0$ then $v\left(\alpha\left(t^{\prime-1}\right)\right)>0($ since $\langle y, \alpha\rangle \neq 0,\langle y, \alpha\rangle \leq 0)$; hence, $v\left(1-\alpha\left(t^{\prime-1}\right)\right)=0$ and $\left|1-\alpha\left(t^{\prime-1}\right)\right|=1$ as required. If $v\left(\alpha\left(t^{\prime-1}\right)\right)=0$ then $\alpha\left(t^{\prime-1}\right)-1 \in \mathcal{O}-\mathfrak{p}$; hence, $v\left(1-\alpha\left(t^{\prime-1}\right)\right)=0$ and $\left|1-\alpha\left(t^{\prime-1}\right)\right|=1$ as required.) Now, we have $t^{\prime-1} g t^{\prime} g^{-1} \equiv z^{-1}\left(\bmod T_{n} B_{n+1}\right)$.

Writing $\operatorname{Ad}(g)\left(t^{\prime} z\right)=t^{\prime} \cdot\left(t^{\prime-1} g t^{\prime} g^{-1}\right) \cdot\left(g z g^{-1}\right)$, we observe that $g z g^{-1} \equiv z$ $\left(\bmod B_{n+1}\right)$ and $t^{\prime-1} g t^{\prime} g^{-1} z \in T_{n} B_{n+1}$; hence, $\operatorname{Ad}(g)\left(t^{\prime} z\right)$ can be written as $t^{\prime} t^{\prime \prime} z^{\prime}$ with $t^{\prime \prime} \in T_{n}$ and $z^{\prime} \in B_{n+1}$.

Lemma 5.3. $B_{1} t B_{1} \subset{ }^{B_{1}}\left(t T_{1}\right)$.

Proof. It is enough to show that $t B_{1} \subset{ }^{B_{1}}\left(t T_{1}\right)$. Let $t_{0} z_{1} \in t B_{1}$ with $t_{0}=t$ and $z_{1} \in$ $B_{1}$. We will construct inductively sequences $g_{1}, g_{2}, \ldots, t_{1}, t_{2}, \ldots$, and $z_{1}, z_{2}, \ldots$ such that $\operatorname{Ad}\left(g_{k} \cdots g_{2} g_{1}\right)\left(t_{0} z_{1}\right)=\operatorname{Ad}\left(g_{k}\right)\left(t_{0} t_{1} \cdots t_{k-1} z_{k}\right)=\left(t_{0} t_{1} \cdots t_{k}\right) z_{k+1}$ with $g_{i} \in B_{i}, t_{i} \in T_{i}$, and $z_{i} \in B_{i}$. 
Applying Lemma 5.2 to $n=1, t^{\prime}=t_{0}$, and $z=z_{1}$, we find $t_{1} \in T_{1}$ and $z_{2} \in B_{2}$ such that $g_{1} t_{0} z_{1} g_{1}^{-1}=t_{0} t_{1} z_{2}$ with $t_{1} \in T_{1}$ and $z_{2} \in B_{2}$. Suppose we found $g_{i} \in B_{i}$, $z_{i+1} \in B_{i+1}$, and $t_{i} \in T_{i}$ for $i=1, \ldots, k$ where $k \geq 1$. Applying Lemma 5.2 to $n=k+1, t^{\prime}=t_{0} t_{1} \cdots t_{k}$, and $z=z_{k+1}$, we find $g_{k+1} \in B_{k+1}, t_{k+1} \in T_{k+1}$, and $z_{k+2} \in$ $B_{k+2}$ so that $g_{k+1} t_{0} t_{1} \cdots t_{k} z_{k+1} g_{k+1}^{-1}=\operatorname{Ad}\left(g_{k+1} \cdots g_{2} g_{1}\right)\left(t_{0} z_{1}\right)=t_{0} t_{1} t_{2} \cdots t_{k+1} z_{k+2}$. (To apply Lemma 5.2 we note that $t^{\prime} \in T(K)_{y}^{\bullet}$ since $t_{0} \in T(K)_{y}^{\bullet}$ and $t_{1} \cdots t_{k} \in T_{1}$, so that for any $\alpha \in R$ we have $\alpha\left(t_{1} \cdots t_{k}\right) \in 1+\mathfrak{p}$.) Taking $g \in B_{1}$ to be the limit of $g_{k} \cdots g_{2} g_{1}$ as $k \rightarrow \infty$, we have $\operatorname{Ad}(g)\left(t_{0} z_{1}\right) \in t T_{1}$.

5.4. Continuing with the proof of Theorem 4.3, we note that by Lemma 5.3 and (5), for the characteristic function $f_{t}$ of $B_{1} t B_{1}$, we have

$$
\operatorname{tr}\left(\sigma_{f_{t}}\right)=\int_{G} f_{t}(g) \phi_{V}(g) d g=\int_{B_{1} t B_{1}} \phi_{V}(t) d g=\operatorname{vol}\left(B_{1} t B_{1}\right) \phi_{V}(t) .
$$

Thus it remains to show

$$
\frac{\operatorname{tr}\left(\sigma_{f_{t}}\right)}{\operatorname{vol}\left(B_{1} t B_{1}\right)}=\frac{\operatorname{tr}\left(\sigma_{\mathfrak{T}_{y}}\right)}{\operatorname{vol}(B t B)}
$$

Since $B_{1}$ is normalized by $B, B$ acts on $V^{B_{1}}$; moreover, since $V$ is irreducible and $V^{B} \neq 0, B$ acts trivially on $V^{B_{1}}$. (Otherwise, there would exist a nonzero subspace of $V$ on which $B$ acts through a nontrivial character of $B / B_{1}$; since $V^{B} \neq 0$, we see that $(V, \sigma)$ would have two distinct cuspidal supports, a contradiction.) Thus we have $V^{B_{1}}=V^{B}$. Since $\sigma_{f_{t}}$ and $\sigma_{\mathfrak{T}_{y}}$ have images contained in $V^{B_{1}}=V^{B}$, it is enough to show

$$
\frac{\operatorname{tr}\left(\left.\sigma_{f_{t}}\right|_{V^{B}}\right)}{\operatorname{vol}\left(B_{1} t B_{1}\right)}=\frac{\operatorname{tr}\left(\left.\sigma_{\mathfrak{T}_{y}}\right|_{V^{B}}\right)}{\operatorname{vol}(B t B)} .
$$

We can find a finite subset $L$ of $T(K)_{0}$ such that $B t B=\bigsqcup_{\tau \in L} B_{1} t B_{1} \tau$. It follows that

$$
\operatorname{vol}(B t B)=\operatorname{vol}\left(B_{1} t B_{1}\right) \sharp(L)
$$

and $\sigma_{\mathfrak{T}_{y}}=\sum_{\tau \in L} \sigma_{f_{t}} \sigma(\tau)$ as linear maps $V \rightarrow V$. Restricting this equality to $V^{B}$ and using the fact that $\sigma(\tau)$ acts as identity on $V^{B}$, we obtain

$$
\left.\sigma_{\mathfrak{T}_{y}}\right|_{V^{B}}=\left.\sharp(L) \sigma_{f_{t}}\right|_{V^{B}}
$$

as linear maps $V^{B} \rightarrow V^{B}$. Clearly, (6) follows from (7) and This completes the proof of Theorem 4.3.

The following result will not be used in the rest of the paper:

Proposition 5.5. If $y \in Y^{++}$and $t \in T(K)_{y}$ then $B t B \subset{ }^{B_{1}} T(K)_{y}$.

Proof. It is enough to show that $t z \subset{ }^{B_{1}} T(K)_{y}$ for any $z \in B$. We can write $z=t_{0} z^{\prime}$, where $t_{0} \in T(K)_{0}, z^{\prime} \in B_{1}$. We have $t z=t t_{0} z^{\prime}$, where $t t_{0} \in T(K)_{y}=T(K)_{y}$ (here we use that $\left.y \in Y^{++}\right)$. Using Lemma 5.3, we have $t t_{0} z^{\prime} \in{ }^{B_{1}}\left(t t_{0} T_{1}\right) \subset{ }^{B_{1}} T(K)_{y}$. 
5.6. In the remainder of this section we assume that $G$ is adjoint. In this case, the irreducible representations $(V, \sigma)$ as in 4.2 (up to isomorphism) are known to be in bijection with the irreducible finite-dimensional representations of the Hecke algebra $H$ (see [Borel 1976]) by $(V, \sigma) \mapsto V^{B}$. The irreducible finitedimensional representations of $H$ have been classified in [Kazhdan and Lusztig 1987] in terms of geometric data; moreover, in [Lusztig 2010], an algorithm to compute the dimensions of the (generalized) weight spaces of the action of the commutative semigroup $\left\{\mathfrak{T}_{y}: y \in Y^{+}\right\}$on any tempered $H$ module is given. In particular the right hand side of the equality in Theorem 4.3 (hence also $\phi_{V}(t)$ in that theorem) is computable when $V$ is tempered.

\section{References}

[Adler and Korman 2007] J. D. Adler and J. Korman, "The local character expansion near a tame, semisimple element”, Amer. J. Math. 129:2 (2007), 381-403. MR 2008a:22020 Zbl 1128.22008

[Borel 1976] A. Borel, "Admissible representations of a semi-simple group over a local field with vectors fixed under an Iwahori subgroup", Invent. Math. 35 (1976), 233-259. MR 56 \#3196 Zbl 0334.22012

[Casselman 1973] W. Casselman, "The Steinberg character as a true character", pp. 413-417 in Harmonic analysis on homogeneous spaces (Williamstown, MA, 1972), edited by C. C. Moore, Amer. Math. Soc., Providence, R.I., 1973. MR 49 \#3039 Zbl 0289.22017

[Casselman 1977] W. Casselman, "Characters and Jacquet modules", Math. Ann. 230:2 (1977), 101-105. MR 58 \#11237 Zbl 0337.22019

[Harish-Chandra 1973] Harish-Chandra, "Harmonic analysis on reductive p-adic groups", pp. 167192 in Harmonic analysis on homogeneous spaces (Williamstown, MA, 1972), edited by C. C. Moore, Amer. Math. Soc., Providence, R.I., 1973. MR 49 \#5238 Zbl 0289.22018

[Iwahori and Matsumoto 1965] N. Iwahori and H. Matsumoto, "On some Bruhat decomposition and the structure of the Hecke rings of p-adic Chevalley groups", Inst. Hautes Études Sci. Publ. Math. 25 (1965), 5-48. MR 32 \#2486 Zbl 0228.20015

[Kazhdan and Lusztig 1987] D. Kazhdan and G. Lusztig, "Proof of the Deligne-Langlands conjecture for Hecke algebras”, Invent. Math. 87:1 (1987), 153-215. MR 88d:11121 Zbl 0613.22004

[Kazhdan and Lusztig 1988] D. Kazhdan and G. Lusztig, "Fixed point varieties on affine flag manifolds”, Israel J. Math. 62:2 (1988), 129-168. MR 89m:14025 Zbl 0658.22005

[Lusztig 2010] G. Lusztig, "Graded Lie algebras and intersection cohomology", pp. 191-224 in Representation theory of algebraic groups and quantum groups, edited by A. Gyoja et al., Progr. Math. 284, Birkhäuser, New York, 2010. MR 2011m:20106 Zbl 1231.20044

[Matsumoto 1969] H. Matsumoto, "Fonctions sphériques sur un groupe semi-simple $p$-adique", C. R. Acad. Sci. Paris Sér. A-B 269 (1969), 829-832. In French. MR 41 \#8576 Zbl 0189.44802

[Moy and Prasad 1994] A. Moy and G. Prasad, "Unrefined minimal $K$-types for $p$-adic groups", Invent. Math. 116:1-3 (1994), 393-408. MR 95f:22023 Zbl 0804.22008

[Rodier 1986] F. Rodier, "Sur le caractère de Steinberg", Compositio Math. 59:2 (1986), 147-149. In French. MR 87m:22045 Zbl 0609.20025

[Shalika 1970] J. A. Shalika, "On the space of cusp forms of a $\mathcal{P}$-adic Chevalley group", Ann. of Math. (2) 92 (1970), 262-278. MR 42 \#423 Zbl 0217.37001 
[Steinberg 1951] R. Steinberg, "A geometric approach to the representations of the full linear group over a Galois field”, Trans. Amer. Math. Soc. 71 (1951), 274-282. MR 13,317d Zbl 0045.30201

Received August 2, 2012. Revised November 26, 2012.

JU-LEE KIM

DEPARTMENT OF MATHEMATICS

MASSACHUSETTS InSTITUTE OF TECHNOLOGY

CAMBRIDGE, MA 02139

UNITED STATES

julee@math.mit.edu

GEORGE LUSZTIG

DEPARTMENT OF MATHEMATICS

MassachusetTs Institute of TeChNOLOGY

CAMBRIDGE, MA 02139-4307

UNITED STATES

gyuri@math.mit.edu 


\title{
PACIFIC JOURNAL OF MATHEMATICS
}

\author{
msp.org/pjm
}

Founded in 1951 by E. F. Beckenbach (1906-1982) and F. Wolf (1904-1989)

\section{EDITORS}

V. S. Varadarajan (Managing Editor)

Department of Mathematics

University of California

Los Angeles, CA 90095-1555

pacific@math.ucla.edu

Paul Balmer

Department of Mathematics

University of California

Los Angeles, CA 90095-1555

balmer@math.ucla.edu

Daryl Cooper

Department of Mathematics

University of California

Santa Barbara, CA 93106-3080 cooper@math.ucsb.edu

Jiang-Hua $\mathrm{Lu}$

Department of Mathematics

Pokfulam Rd., Hong Kong jhlu@maths.hku.hk
The University of Hong Kong

Don Blasius

Department of Mathematics University of California

Los Angeles, CA 90095-1555

blasius@math.ucla.edu

Robert Finn

Department of Mathematics Stanford University

Stanford, CA 94305-2125

finn@math.stanford.edu

Sorin Popa

Department of Mathematics

University of California

Los Angeles, CA 90095-1555 popa@math.ucla.edu

Paul Yang

Department of Mathematics Princeton University

Princeton NJ 08544-1000

yang@math.princeton.edu

\section{PRODUCTION}

Silvio Levy, Scientific Editor, production@msp.org

\section{SUPPORTING INSTITUTIONS}

ACADEMIA SINICA, TAIPEI

CALIFORNIA INST. OF TECHNOLOGY

INST. DE MATEMÁTICA PURA E APLICADA

KEIO UNIVERSITY

MATH. SCIENCES RESEARCH INSTITUTE

NEW MEXICO STATE UNIV.

OREGON STATE UNIV.

\author{
STANFORD UNIVERSITY \\ UNIV. OF BRITISH COLUMBIA \\ UNIV. OF CALIFORNIA, BERKELEY \\ UNIV. OF CALIFORNIA, DAVIS \\ UNIV. OF CALIFORNIA, LOS ANGELES \\ UNIV. OF CALIFORNIA, RIVERSIDE \\ UNIV. OF CALIFORNIA, SAN DIEGO \\ UNIV. OF CALIF., SANTA BARBARA
}

\author{
Vyjayanthi Chari \\ Department of Mathematics \\ University of California \\ Riverside, CA 92521-0135 \\ chari@math.ucr.edu \\ Kefeng Liu \\ Department of Mathematics \\ University of California \\ Los Angeles, CA 90095-1555 \\ liu@math.ucla.edu \\ Jie Qing \\ Department of Mathematics \\ University of California \\ Santa Cruz, CA 95064 \\ qing@cats.ucsc.edu
}

These supporting institutions contribute to the cost of publication of this Journal, but they are not owners or publishers and have no responsibility for its contents or policies.

See inside back cover or msp.org/pjm for submission instructions.

The subscription price for 2013 is US \$400/year for the electronic version, and \$485/year for print and electronic.

Subscriptions, requests for back issues and changes of subscribers address should be sent to Pacific Journal of Mathematics, P.O. Box 4163, Berkeley, CA 94704-0163, U.S.A. The Pacific Journal of Mathematics is indexed by Mathematical Reviews, Zentralblatt MATH, PASCAL CNRS Index, Referativnyi Zhurnal, Current Mathematical Publications and the Science Citation Index.

The Pacific Journal of Mathematics (ISSN 0030-8730) at the University of California, c/o Department of Mathematics, 798 Evans Hall \#3840, Berkeley, CA 94720-3840, is published twelve times a year. Periodical rate postage paid at Berkeley, CA 94704, and additional mailing offices. POSTMASTER: send address changes to Pacific Journal of Mathematics, P.O. Box 4163, Berkeley, CA 94704-0163.

PJM peer review and production are managed by EditFLOW ${ }^{\circledR}$ from Mathematical Sciences Publishers.

\section{PUBLISHED BY}

mathematical sciences publishers

nonprofit scientific publishing

http://msp.org/

(C) 2013 Mathematical Sciences Publishers 


\section{PACIFIC JOURNAL OF MATHEMATICS}

Volume $265 \quad$ No. $2 \quad$ October 2013

Singularity removability at branch points for Willmore surfaces

YANN BERNARD and TRISTAN RIVIÈRE

On Bach flat warped product Einstein manifolds

QIANG CHEN and CHENXU HE

On plane sextics with double singular points

Alex DeGTyareV

A computational approach to the Kostant-Sekiguchi correspondence

HEIKo DiETRICH and WILLEM A. DE GRAAF

Landau-Toeplitz theorems for slice regular functions over quaternions

GRAZIANO GENTILI and GiUlia SARFATTI

On surgery curves for genus-one slice knots

PATRICK M. GILMER and CHARLES LIVINGSTON

Characterizing abelian varieties by the reduction of the Mordell-Weil group

Chris Hall and Antonella Perucca

Variation of complex structures and the stability of Kähler-Ricci

Solitons

StUART J. HALl and ThOMAS MURPHY

On crossed homomorphisms of the volume preserving diffeomorphism groups

RYOJI KASAGAWA

Regularity at the boundary and tangential regularity of solutions of the Cauchy-Riemann system

TRAN VU KHANH and GIUSEPPE ZAMPIERI

On the Steinberg character of a semisimple $p$-adic group 